\title{
Satisfação e sobrecarga de profissionais de saúde mental: validade de construto das escalas SATIS-BR e IMPACTO-BR
}

Satisfaction and burden of mental health professionals:
construct validity of the scales SATIS-BR and IMPACTO-BR

Marina Bandeira', Sergio Ishara², Antonio Waldo Zuardi²

\section{RESUMO}

Objetivo: Os níveis de satisfação e sobrecarga de profissionais em serviços de saúde mental afetam tanto a sua saúde e o bem-estar quanto a qualidade do cuidado prestado aos pacientes. Para avaliar estas dimensões, é necessário ter acesso a escalas de medida válidas e fidedignas. Este artigo visou descrever um estudo da validade de construto de duas escalas de avaliação da satisfação (SATIS-BR) e da sobrecarga (IMPACTO-BR) de profissionais em serviços de saúde mental. Métodos: As escalas foram aplicadas a uma amostra de 172 profissionais, que trabalhavam em seis serviços de psiquiatria de uma cidade de porte médio de São Paulo. A validade de construto foi avaliada a partir do teste da hipótese de que deve haver uma correlação significativa entre dois construtos teoricamente relacionados. Para isso, foram feitas análises estatísticas correlacionais de Spearman entre as escalas de satisfação e sobrecarga, assim como uma comparação de dois subgrupos de sujeitos, com e sem indicativo de estresse, com relação aos seus graus de satisfação e sobrecarga no trabalho. Resultados: Obteve-se correlação significativa negativa esperada entre os escores de satisfação e sobrecarga $(r=-0,38)$. Obteve-se ainda a diferença significativa entre dois

\section{Palavras-chave}

Fidedignidade e validade, escalas de satisfação e sobrecarga da equipe, avaliação de serviços da saúde mental. grupos de sujeitos, com e sem estresse, com relação ao grau de satisfação e sobrecarga no trabalho, sujeitos com estresse apresentando níveis mais baixos de satisfação no trabaIho e grau mais elevado de sobrecarga. Conclusões: Conclui-se que as escalas analisadas apresentam validade de construto e são adequadas para monitorar a qualidade de serviços de saúde mental. A escala de sobrecarga constituiu um preditor do estresse apresentado pelos trabalhadores em saúde mental.

\section{ABSTRACT}

Objective: Satisfaction and burden of professionals in mental health services can affect their well being and also the quality of the care they provide to the patients. In order to evaluate those dimensions, it is necessary to have access to valid and reliable measure scales. This study describes

As escalas podem ser acessadas no site www.lapsam.ufsj.edu.br

1 Laboratório de Pesquisa em Saúde Mental (Lapsam). Departamento de Psicologia. Universidade Federal de São João del Rei (UFSJ). 


\section{Key-words}

Mental health services, satisfaction and burden, professional team, service evaluation. the evaluation of the construct validity of two scales measuring satisfaction (SATIS-BR) and burden (IMPACTO-BR) of professionals in mental health services. Methods: The scales were applied to a sample of 172 professionals who worked in six mental health services in a medium city of the state of São Paulo. Construct validity was evaluated by testing the hypotheses that theoretically related constructs should present statistical significant correlation. A Spearman correlation analysis was performed between those two scales and a comparison was made between two groups of subjects, with and without indicative of stress, regarding their levels of satisfaction and burden. Results: A significant negative correlation was observed between the satisfaction and burden scores ( $r=-0,38)$. It was also observed a significant difference in satisfaction and burden degree between the two groups of subjects, with and without indicative of stress, subjects with stress presenting lower degree of job satisfaction and higher level of burden in work. Conclusions: It was concluded that the satisfaction and burden scales analyzed present construct validity and are adequate to evaluate the quality of mental health services. Burden scores were predictive of stress in mental health professionals.

\section{INTRODUÇÃO}

A avaliação dos serviços de saúde mental tem sido recomendada como um procedimento permanente, sendo necessário para promover a qualidade dos serviços, o que tem sido uma preocupação constante da Organização Mundial de Saúde (OMS) (1996). Uma avaliação integrativa tem sido estimulada, incluindo a participação do conjunto das pessoas envolvidas nestes serviços: pacientes, familiares e profissionais. A avaliação recomendada tem como objetivo estimar o nível de satisfação dos três segmentos e o grau de sobrecarga de familiares e profissionais dos serviços (1996). A apreciação dos pacientes e familiares e dos profissionais são duas perspectivas diferentes, porém complementares e igualmente necessárias a uma avaliação completa da qualidade destes serviços (Bandeira et al., 1999 e 2000). Esta avaliação fornece informações essenciais que permitem aos serviços fazer a constante reavaliação dos seus procedimentos de atendimento, identificar possíveis deteriorações na qualidade do serviço e modificar seus procedimentos, visando melhorar a qualidade (Bandeira et al., 2000).

A satisfação do profissional nos serviços envolve um sentimento positivo resultante da avaliação de sua experiência de trabalho, conforme suas metas e valores pessoais (Lino, 2004). No âmbito do estudo multicêntrico da OMS, a satisfação dos profissionais de saúde mental foi definida em relação às seguintes dimensões: condições de trabalho, relacionamento com os colegas, qualidade do tratamento oferecido aos pacientes, participação e envolvimento na equipe (World Health Organization, 1996). O conceito de sobrecarga se refere à percepção de demandas excessivas para a pessoa e ao sentimento de ter um peso a carregar em conseqüência destas demandas (Maurin e Boyd, 1990). No caso dos profissionais de saúde mental, esta percepção resulta do contato contínuo com pessoas portadoras de distúrbios psiquiátricos, incluindo sentimentos negativos, como frustração com os resultados do trabalho, cansaço, medo de ser agredido e desejo de mudar de emprego (World Health Organization, 1996). A sobrecarga percebida pela pessoa no seu trabalho pode resultar estresse emocional, definido por Lipp (2000) como uma reação complexa e global do organismo, que ocorre quando a pessoa é exposta a fatores estressantes que ultrapassam sua capacidade de resistir física e emocionalmente, reação que envolve componentes físicos, psicológicos, emocionais e hormonais e que se desenvolve em quatro fases crescentes: alerta, resistência, quase-exaustão e exaustão. De início, o estresse é positivo, energizando e preparando a pessoa para a ação (fase 1), em seguida há tentativa automática de lidar com os estressores para manter a homeostase (fase 2) e, se os estressores persistem em freqüência ou intensidade, há quebra de resistência, primeiro sem afetar totalmente o funcionamento da pessoa (fase 3), mas finalmente resultando exaustão, incapacidade de funcionar e adoecimento (fase 4). As duas últimas fases, portanto, se assemelham, em gravidade, à síndrome do Burnout, que segundo Maslach (2005), envolve três dimensões, que se referem às reações negativas de exaustão emocional, ao ceticismo e ao desligamento do trabalho e à sensação de ineficácia e à falta de realização. Tem sido considerado como uma síndrome resultante de exposição prolongada ao estresse (Hannigan et al., 2000). Como o conceito de burnout se refere a níveis extremos de impacto do trabalho, contrariamente ao conceito de estresse, considerou-se, no presente trabalho, mais pertinente investigar a relação entre os conceitos de sobrecarga e estresse.

Baixos níveis de satisfação com o serviço e a sobrecarga sentida pelos profissionais em decorrência do trabalho 
diário com portadores de transtornos mentais podem afetar seu bem-estar e saúde mental e podem ter conseqüências na qualidade dos cuidados prestados aos pacientes e, portanto, na qualidade dos serviços. Tem sido observado, em diversas organizações, que há uma relação entre a satisfação do profissional e a qualidade do serviço prestado ao usuário ou consumidor (Farrel e Dares, 1999). Wykes et al. (1997) enfatizaram a necessidade de avaliar as repercussões do trabalho em saúde mental na qualidade de vida destes profissionais e de considerar estas dimensões ao se avaliar os custos, a viabilidade e a qualidade dos serviços de saúde mental, uma vez que a baixa satisfação e a sobrecarga sentida pelos profissionais podem afetar o funcionamento destes serviços.

Gilman e Diamond (1985) apontam a necessidade de avaliar os custos dos serviços de saúde mental, além da estimativa da sua eficácia clínica. Os autores citam os custos econômicos diretos (medicamentos, alimentação, moradia, serviços médicos gerais etc.) ou indiretos (perda de produtividade), mas também os seus custos não-econômicos, como o sofrimento ou a sobrecarga sentida pelos profissionais das equipes de trabalho. Além de contribuir para os custos de um serviço, os sentimentos de insatisfação e sobrecarga dos profissionais podem afetar, de acordo com estes autores, a própria eficácia do tratamento fornecido aos pacientes.

Diversos estudos foram realizados para investigar o grau de sobrecarga de profissionais de saúde em geral e de saúde mental, em particular. Aronson (2005) encontrou, em trabalhadores de hospitais psiquiátricos privados, menores níveis de satisfação entre enfermeiros do que em outras categorias profissionais, principalmente em enfermeiros em posição de supervisores. Robinson et al. (2003) identificaram altos níveis de exaustão emocional em enfermeiros, associada à elevada responsabilidade e à carga de trabalho.

Uma comparação entre serviços mostrou que havia maior grau de exaustão emocional em enfermeiros de serviços de saúde mental do que em enfermeiros de serviços de saúde em geral (Hannigan et al., 2000) ou entre aqueles que atuavam na área forense (Happell et al., 2003). Hannigan et al. (2000) observaram alto nível de exaustão emocional entre enfermeiros que trabalhavam em serviços de saúde mental comunitária, o que se confirmou no estudo de Wykes et al. (1997), que também observaram este mesmo resultado em equipes diversificadas de profissionais de seis serviços comunitários de saúde mental.

Diversos fatores foram associados aos níveis de satisfação e sobrecarga dos profissionais de saúde mental. Um destes fatores consiste no estado de gravidade do doente mental, segundo pesquisa de Acker (1999), realizada sobre a satisfação profissional de assistentes sociais da área de saúde mental. Farrell e Dares (1999) observaram, em estudo com enfermeiros, que as características mais importan- tes para a satisfação dos profissionais foram: interesse pelo trabalho, relacionamento com os colegas, ter responsabilidades e independência. Os motivos referidos para a diminuição da satisfação foram: a falta de oportunidade para o desenvolvimento profissional e a falta de autonomia e valorização. Receber apoio no trabalho constituiu-se uma fonte de satisfação destacada por Reid et al. (1999), visto que o contato com os colegas foi a fonte mais freqüente de satisfação. Gulliver et al. (2003) encontraram uma relação entre a satisfação dos membros da equipe e a organização do trabalho, seu nível de conquista e a relação com outros membros da equipe.

Na pesquisa de Wykes et al. (1997), avaliando equipes profissionais de atendimento comunitário de saúde mental, foram observados altos níveis de exaustão emocional e despersonalização, superiores aos encontrados em estudos com profissionais dos hospitais, porém os escores referentes à falta de realização no trabalho foram menos elevados. Os resultados foram relacionados principalmente às dificuldades cotidianas do trabalho (hastles) e ao local de trabalho. Observaram-se ainda níveis elevados de ansiedade e um terço da equipe apresentou escores, em uma escala de saúde geral, que se situavam acima do nível considerado sintomático de transtornos psicológicos. Os resultados apontaram para a dificuldade particular do modelo comunitário de saúde mental, resultante da desinstitucionalização psiquiátrica, que requer novas demandas dos profissionais e uma nova organização do trabalho, envolvendo maior interação face a face com os pacientes, visitas domiciliares em áreas isoladas, falta de segurança e pouco apoio de colegas (Wykes et al., 1997). No estudo de Hannigan et al. (2000), com profissionais de serviços comunitários de saúde mental, os aspectos destacados foram a falta de estabilidade no trabalho e a carência de apoio institucional.

A avaliação da satisfação e da sobrecarga sentidas pelos profissionais de saúde mental requer, entretanto, o acesso a instrumentos de medida válidos e fidedignos, capazes de avaliar estas dimensões. Segundo Ruggeri (1994), em geral tem sido negligenciada a análise das qualidades psicométricas dos instrumentos de medida em saúde mental, o que compromete a validade dos resultados e dificulta a comparabilidade dos estudos.

O objetivo do presente estudo foi avaliar a validade de construto de duas escalas que medem a satisfação e a sobrecarga de profissionais de saúde mental, elaboradas pela OMS e adaptadas e validadas para o Brasil por Bandeira et al. (1999 e 2000). A validade de construto dessas escalas foi avaliada pelo procedimento de análise de hipótese, consoante a qual uma escala de medida deve correlacionar significativamente com outras variáveis relacionadas ao construto medido por ela (Pasquali, 2003). 


\section{MÉTODO}

\section{Participantes}

Participaram do estudo 172 sujeitos, sendo 136 profissionais contratados e 36 médicos residentes de psiquiatria, que trabalhavam em seis serviços de psiquiatria de uma cidade de porte médio. Dentre estes, três serviços eram de um hospital universitário, sendo um hospital-dia, uma enfermaria e uma enfermaria de internação breve. As outras três unidades foram: um centro de atenção psicossocial e duas unidades de um hospital psiquiátrico, uma masculina e uma feminina.

A amostra constituiu-se de quase a totalidade dos profissionais dos serviços analisados, com exceção de dois sujeitos que foram excluídos por terem respondido apenas parcialmente aos questionários e dez que não chegaram a ser entrevistados por motivo de férias ou falta ao trabalho.

\section{Instrumentos de medida}

Na coleta de dados foram utilizados três instrumentos: a Escala de Avaliação da Satisfação de Profissionais em Serviços de Saúde Mental (SATIS-BR), a Escala de Avaliação da Sobrecarga de Profissionais em Serviços de Saúde Mental (IMPACTO-BR) e o Inventário de Sintomas de Estresse de Lipp (ISSL).

As escalas SATIS-BR e IMPACTO-BR integram um conjunto de escalas para avaliação de serviços de saúde mental, elaboradas pela Divisão de Saúde Mental da OMS, que foram validadas para o Brasil por Bandeira et al. (1999 e 2000).

\section{Escala SATIS-BR}

Esta escala avalia o grau de satisfação da equipe de profissionais em serviços de saúde mental. O questionário completo contém 69 questões, dentre as quais apenas 32 constituem as questões quantitativas da escala propriamente dita que avaliam o grau de satisfação. A escala inclui alternativas de respostas dispostas em escala ordinal tipo Likert de 5 pontos, em que 1 = muito insatisfeito e 5 = muito satisfeito. As demais questões são descritivas ou qualitativas e avaliam as condições de trabalho dos profissionais e dados sociodemográficos. Algumas questões avaliavam as variáveis sociodemográficas.

Esta escala é composta por quatro subescalas que avaliam diferentes dimensões da satisfação com o serviço. A primeira subescala inclui 10 questões que avaliam o grau de satisfação da equipe em relação à qualidade do serviço oferecido aos pacientes. O estudo da consistência interna obteve, para esta subescala, um valor de alfa de Cronbach de 0,83 e as correlações item-total variaram de 0,36 a 0,73. A segunda subescala inclui 7 questões que avaliam o grau de satisfação da equipe em relação à sua participação no serviço (nas decisões, na implantação dos tratamentos e na avaliação do serviço, na expressão de suas opiniões etc.).
Esta subescala apresentou valor de alfa de 0,72 e as correlações item-total variaram de 0,17 a 0,70.

A terceira subescala inclui 10 questões que avaliam o grau de satisfação da equipe em relação às condições gerais de trabalho (instalações, salário, benefícios, conforto e a aparência do serviço, medidas de segurança e confidencialidade, clima do ambiente de trabalho). O valor de alfa foi de 0,77 e as correlações item-total variaram entre 0,26 a 0,56. A quarta subescala inclui três questões que avaliam o grau de satisfação do profissional a respeito do seu relacionamento com os colegas de trabalho e com os seus superiores, assim como sua satisfação com o grau de sua autonomia em relação às outras pessoas no serviço. O valor de alfa para esta subescala foi de 0,63 e as correlações item-total variaram entre 0,36 e 0,53.

A escala global consta do total de 32 itens, incluindo os 30 itens que compõem as subescalas e mais 2 itens que se referem à satisfação da equipe com a freqüência de contatos entre pacientes e profissionais. A escala global apresenta elevada consistência interna, com o valor de alfa igual a 0,89 e correlações item-total variando entre 0,24 e 0,74.

\section{Escala IMPACTO-B}

Esta escala avalia a sobrecarga sentida pelos profissionais de saúde mental, em conseqüência do trabalho diário com pessoas portadoras de distúrbios psiquiátricos. O questionário completo inclui 34 questões, dentre as quais apenas 18 constituem as questões quantitativas da escala propriamente dita, avaliando as repercussões do trabalho em diversos aspectos da vida do profissional. As alternativas de resposta estão dispostas em escala tipo Likert de 5 pontos, em que 1 = de forma alguma e 5 = extremamente. As demais questões são descritivas e avaliam as condições de trabalho e dados sociodemográficos.

Os itens desta escala se distribuem em três subescalas. A primeira contém 5 itens que avaliam o impacto do trabalho sobre a saúde física e mental do profissional (freqüência de problemas físicos, de consultas ao médico, de tomar medicamentos, efeitos na estabilidade emocional etc.). $O$ estudo da consistência interna obteve, para esta subescala, valor de alfa igual a 0,78 e correlações item-total variando de 0,27 a 0,77 .

A segunda subescala contém 6 itens que avaliam o impacto da sobrecarga ressentida no trabalho sobre o funcionamento dos profissionais (freqüência em que pensa em mudar de campo de trabalho, que outro tipo de trabalho o faria sentir-se mais saudável, afastamentos, receio de ser agredido, qualidade do sono, saúde em geral). Esta subescala apresentou valor de alfa igual a 0,77 e correlações itemtotal entre 0,40 e 0,63. A terceira subescala contém 5 itens que avaliam os efeitos do trabalho no estado emocional do profissional, no seu sentimento de estar sobrecarregado (sentir-se frustrado, cansado, sobrecarregado, deprimido e 
estressado por trabalhar com doença mental). O valor de alfa obtido para esta subescala foi de 0,70 e as correlações item-total variaram de 0,32 a 0,68.

A escala global contém o total de 18 itens, os quais incluem as 16 questões dos três fatores descritos anteriormente e duas questões adicionais que apresentaram correlações item-total de 0,48 e 0,55. Estas duas questões se referem ao impacto do trabalho em saúde mental sobre a vida social do profissional e sobre sua vida familiar. O valor do coeficiente alfa de Cronbach para a escala global foi de 0,87 e as correlações item-total variaram de 0,24 a 0,7.

\section{Inventário de sintomas de estresse para adultos de Lipp (ISSL)}

Esta escala foi inicialmente elaborada e validada por Lipp e Guevara, em 1994, e posteriormente por Lipp (2000). A escala apresenta uma avaliação de sintomas de estresse, tendo como objetivo verificar a ocorrência de estresse e, em caso positivo, indicar a fase e o tipo de sintomatologia predominante. $O$ protocolo de avaliação encontra-se dividido em quatro fases correspondentes às fases do estresse: alerta, resistência, quase exaustão e exaustão. Solicita-se ao participante que assinale, na primeira parte, os sintomas que apresentou nas últimas 24 horas, a partir de uma lista com 15 sintomas de estresse. A segunda parte apresenta 15 itens e corresponde aos sintomas experimentados na última semana. A terceira parte está composta por 23 sintomas, dos quais devem ser assinalados aqueles experimentados no último mês.

\section{Procedimento}

Foi solicitada a colaboração de cada participante, destacando-se ser esta voluntária e sigilosa. Os participantes foram informados sobre a natureza da pesquisa, os objetivos, os procedimentos utilizados e a ausência de riscos e danos decorrentes da participação no estudo, a possibilidade de desistência a qualquer momento, sem nenhum ônus para seu trabalho, bem como a garantia de sigilo dos dados obtidos e da disponibilidade do pesquisador para qualquer esclarecimento necessário. Todos os que concordaram em participar da pesquisa assinaram o Termo de Consentimento Informado. O estudo recebeu autorização de cada um dos serviços para sua realização e aprovação do Comitê de Ética do Hospital das Clínicas da Faculdade de Medicina de Ribeirão Preto - USP.

Os questionários foram aplicados por um dos autores (S.I.) e envolveram a coleta de dados em diferentes dias e horários de acordo com a disponibilidade dos participantes. Os questionários foram sempre respondidos de maneira individual e quando possível, a aplicação foi realizada para vários profissionais simultaneamente. Os instrumentos escolhidos são auto-aplicáveis, mas a coleta foi acompanhada pelo autor, visando o esclarecimento de eventuais dúvidas.

\section{Análise de dados}

A validade de construto das escalas SATIS-BR e IMPACTOBR foi avaliada pelo procedimento de análise de hipóteses, testando-se a hipótese de que a escala deve correlacionarse significativamente com outras medidas de variáveis que são relacionadas ao construto medido (Pasquali, 2003). Neste procedimento de validação, considera-se que a evidência de associações significativas entre dois instrumentos que avaliam construtos diferentes, mas teórica e empiricamente relacionados, serve como indicador da validade de ambos, indicando que eles estão relacionados, conforme a expectativa.

Medidas de estresse, satisfação e sobrecarga comparadas, pois estes construtos estão relacionados entre si. A sobrecarga sentida pelos profissionais no serviço de saúde mental pode afetar seu bem-estar psicológico e sua saúde mental, podendo elevar seu nível de estresse. Além disso, espera-se que a sobrecarga sentida pelos profissionais no serviço esteja inversamente relacionada ao seu nível de satisfação com o serviço. Essas relações eram investigadas, no presente estudo, por meio de análises estatísticas dos escores obtidos nas escalas de satisfação, sobrecarga e estresse.

Para isso, foram feitas duas análises. A primeira incluiu a análise do coeficiente de correlação de Spearman entre os escores das escalas de satisfação e sobrecarga, visando testar a hipótese de que estavam negativamente correlacionados. A segunda envolveu a comparação entre dois grupos, com e sem indicativo de estresse, medido pela escala ISSL, por meio do teste $t$ de Student, visando testar a hipótese de que apresentariam diferença significativa entre eles, o grupo com indicativo de estresse apresentando menor grau de satisfação e maior grau de sobrecarga.

\section{RESULTADOS}

\section{Descrição da amostra}

Os participantes tinham a idade média de 37,35 anos (d. p. = 10,08), sendo o mínimo de 18 e o máximo de 64 anos. Houve predomínio da participação de mulheres (62,2\%). Em relação ao estado civil, a maioria era de pessoas casadas ou que viviam com um companheiro (45,9\%); em segundo lugar, pessoas solteiras (39,5\%); seguidos de pessoas viúvas e separadas (7,7\%). Em relação ao nível de escolaridade, houve predomínio de profissionais com nível superior $(58,7 \%)$ e nível médio (41,3\%). A maioria dos profissionais era auxiliar de enfermagem ou atendente de enfermagem (37,8\%), médicos residentes (20,9\%) e enfermeiras (11,6\%), seguidos de psicólogos, terapeutas ocupacionais e assistentes sociais (11,0\%), técnicos $(11,0 \%)$ e médicos contratados (7,6\%). Observou-se predominância de profissionais que trabalhavam há mais de dez anos na área de saúde mental (37,8\%) ou 
entre 1 a 6 anos (36,0\%) e, em seguida, entre 6 e 10 anos (17,40\%) e menos de 1 ano (11\%).

\section{Relação entre satisfação e sobrecarga}

Obteve-se a correlação negativa significativa entre os escores de satisfação e de sobrecarga $(r=-0,38 ; p<0,01)$. Assim, os escores de satisfação estavam inversamente associados aos escores de sobrecarga, ou seja, quanto maior era o grau de sobrecarga sentida pelos profissionais, menor era o seu nível de satisfação no trabalho.

\section{Diferença entre grupos com e sem estresse}

Foram comparados dois subgrupos de sujeitos da amostra, classificados segundo a presença e a ausência de estresse, com base na escala ISSL e os resultados estão apresentados na Tabela 1. Observou-se diferença significativa entre os dois grupos com relação ao grau de satisfação ( $t=3,96$; $p<0,001$ ), tendo o grupo com estresse apresentado menor grau de satisfação com o serviço do que o grupo sem estresse. Obteve-se igualmente diferença significativa entre os dois grupos no que se refere ao grau sobrecarga $(\mathrm{t}=$ 9,79; $p<0,001)$, tendo-se observado escore mais elevado de sobrecarga no serviço no grupo de profissionais que apresentavam indicativo de estresse.

Tabela 1. Níveis de satisfação e sobrecarga em termos de média (M) e desvio-padrão (DP), nos grupos com e sem manifestações de estresse

\begin{tabular}{llccccc}
\hline & & \multicolumn{3}{c}{ Satisfação } & & \multicolumn{2}{c}{ Sobrecarga } \\
\cline { 3 - 4 } \cline { 7 - 8 } & & $M$ & $D P$ & & $M$ & DP \\
\hline Estresse (ISSL) & Presente & $2,97^{*}$ & 0,49 & & $2,67^{*}$ & 0,71 \\
& Ausente & 3,33 & 0,55 & & 1,74 & 0,49 \\
\hline
\end{tabular}

${ }^{*} p<0,05$

\section{DISCUSSÃO}

No presente estudo, foi observada correlação negativa significativa entre os escores de satisfação e sobrecarga, bem como níveis mais baixos de satisfação e mais elevados de sobrecarga, nos sujeitos com indicativo de estresse em relação ao grupo sem estresse. Estes resultados confirmam a hipótese de que há uma associação entre estas variáveis, conforme esperado, atestando a validade de construto (Pasquali, 2003) das escalas de satisfação (SATIS-BR) e de sobrecarga (IMPACTO-BR), aplicada aos profissionais de serviços de saúde mental.

As informações obtidas com estas escalas poderão ser importantes como indicadoras da qualidade de determinado serviço de saúde mental, uma vez que a satisfação e a sobrecarga sentidas pelos profissionais das equipes de trabalho podem afetar diretamente a qualidade do atendimento prestado aos pacientes (Chiavenato, 1999; Wykes et al., 1997). Este tipo de avaliação pode indicar a necessidade de se modificar aspectos do funcionamento do serviço ou de implantar procedimentos preventivos de apoio e acompanhamento dos profissionais, por exemplo, em atividades de supervisão. $\mathrm{O}$ apoio aos profissionais pode servir para diminuir a ocorrência de absenteísmo ou de rotatividade, assim como promover a satisfação dos profissionais no seu trabalho.

Pesquisas avaliativas da satisfação e sobrecarga podem ser importantes, ainda, para comparar diferentes tipos de serviços de saúde mental, em termos de seu impacto nos profissionais. Diferentes categorias profissionais podem igualmente ser avaliadas, no interior de um mesmo serviço, podendo identificar necessidades diferenciadas. Estas escalas podem ser úteis, portanto, para avaliar estas diferenças em serviços de saúde mental.

O fato de a amostra utilizada nesta pesquisa apresentar grande ligação com serviços universitários dificulta a generalização dos resultados, constituindo-se uma limitação do estudo. Pesquisas futuras com amostras mais amplas e diversificadas poderão complementar os resultados do presente estudo.

\section{CONCLUSÃO}

Os dados desta pesquisa contribuíram para acrescentar novas informações sobre as propriedades psicométricas das escalas SATIS-BR e IMPACTO-BR, além das obtidas em estudos precedentes (Bandeira et al., 2000). Os resultados mostraram que estas escalas apresentam bons indicadores de validade de construto, pois apresentaram correlações significativas entre variáveis, na direção esperada pelas hipóteses postuladas. Pode-se concluir, portanto, que elas constituem instrumentos de medida adequados para serem utilizados em avaliações do grau de satisfação e sobrecarga dos profissionais nos serviços de saúde mental.

Sendo multifatoriais, estas escalas permitem avaliar, com escores de medida independentes, diferentes dimensões dos serviços, separadamente pelas subescalas. Além disso, estas escalas permitirem obter informações diferenciadas, uma vez que foram construídas para avaliar especificamente serviços de saúde mental. Escalas de medida validadas são necessárias para avaliar, de maneira padronizada estes serviços, permitindo assim a confiabilidade dos dados e a comparabilidade entre os resultados de diferentes pesquisadores (Ruggeri, 1994). Estas escalas constituem um esforço da OMS para fornecer instrumentos padronizados comparáveis para serem usados em diferentes países, na avaliação de serviços de saúde mental.

Conflito de interesse: AWZ possui bolsa de produtividade em pesquisado CNPq (n 300775/2004-0) e da Fapesp (Grant no 2002/13197-2). MB possui bolsa de produtividade do CNPq (no 300265/2004-2). 


\section{REFERÊNCIAS}

Acker GM. The impact of clients' mental illness on social workers' job satisfaction and burnout. Health Soc Work, 24(2):112-9, 1999

Aronson K. Job satisfaction of nurses who work in private psychiatric hospitals. Psychiatr Serv. 56(1):102-4, 2005.

Bandeira M, Pitta AMF, Mercier C. Escalas brasileiras de avaliação da satisfação (SATIS-BR) e da sobrecarga (IMPACTO-BR) da equipe técnica em serviços de saúde mental. J Bras Psiq. 49(4):105-15, 2000.

Bandeira M, Pitta AMF, Mercier C. Escalas da OMS de avaliação da satisfação e da sobrecarga em serviços de saúde mental. J Bras Psiq. 48(6):233-44, 1999.

Chiavenato I. Gestão de pessoas: o novo papel dos recursos humanos nas organizações. Rio de Janeiro: Campus, 1999

Farrel GA, Dares G. Nursing staff satisfaction on a mental health unit. Aust N Z J Ment Health Nurs. 8(2):51-7, 1999.

Gilman DR, Diamond RJ. Economic analysis in community treatment of the chronically mentally ill. In: Stein LI, Test MA. The Training in community living model: a decade of experience. New Directions for Mental Health Services. San Fransisco: Jossey-Bass Publishers, p. $77-84,1985$

Gulliver P, Towell D, PeckE. Staff morale in the merger of mental health and social care organizations in England. J Psychiatr Ment Health Nurs. 10(1):101-7, 2003.

Hannigan B, Edwards D, Coyle D, Fothergill A, Burnard P. Burnout in community mental health nurses: findings from the all-wales stress study. J Psychiatr Ment Health Nurs. 7(2):127-34, 2000.

Happell B, Martin T, Pinikahana J. Burnout and job satisfaction: a comparative study of psychiatric nurses from forensic and a mainstream mental health service. Int J Ment Health Nurs. 12(1):39-47, 2003.
Lipp, MEN. Manual do inventário de sintomas de stress para adultos de Lipp (ISSL). São Paulo. Casa do Psicólogo, 2000. 40 p.

Lino, M. M. Qualidade de vida e satisfação profissional de enfermeiras de unidades de terapia intensiva. 2004. 223 p. [tese de doutorado]. São Paulo : Escola de Enfermagem da Universidade de São Paulo, 2004.

Maurin JT, Boyd CB. Burden of mental illness on the family : a critical review. Archives of Psychiatric Nursing. 4(2):99-107, 1990.

Maslach,C. Entendendo o burnout. In: Rossi AM, Perrewé PL, Sauter SL. Stress e qualidade de vida no trabalho: perspectives atuais. São Paulo: Atlas, p. 41-55, 2005.

Pasquali L. Psicometria: teoria dos testes na psicologia e na educação. Petrópolis, RJ: Vozes, 2003.

Reid Y, Johnson S, Morant N, Kuipers E, Szmukler G, Thornicroft G, et al. Explanations for stress and satisfaction in mental health professionals: a qualitative study. Soc Psychiatry Psychiatr Epidemiol. 34(6):301-8, 1999.

Robinson JR, Clements K, Land C. Workplace stress among psychiatric nurses. Prevalence, distribution, correlates, predictors. J Psychosoc Nurs Ment Health Serv. 41(4):32-41, 2003

Ruggeri M. Patients'and relatives'satisfaction with psychiatric services : the state of the art of its measurement. Soc Psychiatry Psychiatr Epidemiol. 29: 212-27, 1994.

Tummers GE, Janssen PP, Landeweerd A, Houdes I. A comparative study of work characteristics and reactions between general and mental health nurses: a multi-sample analysis. J Adv Nurs. 36(1):151-62, 2001.

World Health Organization. WHO-SATIS Consumer's and caregivers' satisfaction with mental health services: a multisite study. Geneva: Division of Mental Health. WHO, 1996.

Wykes T, Stevens W, Everitt B. Stress in community care teams: will it affect the sustainability of community care? Soc Psychiatry Psychiatr Epidemiol. 32:398-407, 1997. 